\title{
Melioidosis in a patient with type 1 diabetes mellitus on an insulin pump
}

\author{
Melissa Katz', Simon Smith2,3, Luke Conway ${ }^{1,3}$ and Ashim Sinha1,3 \\ 'Department of Diabetes and Endocrinology, Infectious Diseases, Cairns Hospital, Cairns, Queensland, Australia, and \\ 3School of Medicine and Dentistry, James Cook University, Cairns, Queensland, Australia
}

Correspondence should be addressed to M Katz

Email

melissathomas105@gmail. com

\section{Summary}

Diabetes mellitus is a well-recognised risk factor for melioidosis, the disease caused by Burkholderia pseudomallei, which is endemic in northern Australia and Southeast Asia. We present the initial diagnostic dilemma of a febrile patient from northern Australia with type 1 diabetes mellitus and negative blood cultures. After a 6-week history of fevers and undifferentiated abdominal pain, MRI of her spine revealed a psoas abscess. She underwent drainage of the abscess which cultured B. pseudomallei. She completed 6 weeks of intravenous (IV) ceftazidime and oral trimethoprim/ sulphamethoxazole (TMP/SMX) followed by a 12-week course of oral TMP/SMX. We postulate that the likely route of infection was inoculation via her skin, the integrity of which was compromised from her insulin pump insertion sites and an underlying dermatological condition.

\section{Learning points:}

- Diabetes mellitus is the strongest risk factor for developing melioidosis.

- Atypical infections need to be considered in individuals with diabetes mellitus who are febrile, even if blood cultures are negative.

- There is heterogeneity in the clinical presentation of melioidosis due to variable organ involvement.

- Consider melioidosis in febrile patients who have travelled to northern Australia, Asia and other endemic areas.

\section{Background}

Melioidosis is an opportunistic infection endemic in the tropics and caused by the environmental bacterium Burkholderia pseudomallei $(1,2)$. Risk factors for the disease include diabetes mellitus (DM), hazardous alcohol use, chronic kidney disease and chronic lung disease. Diabetes mellitus, most commonly type 2 , is the most frequent risk factor, occurring in 40-60\% of patients who are diagnosed with melioidosis $(1,3,4,5)$. Acquisition of melioidosis most often occurs through inoculation, with compromised skin integrity increasing the risk of developing the disease (4). Given the protean clinical manifestations of melioidosis, a high index of suspicion is required to ensure appropriate investigations and empirical management is initiated in those at risk living in endemic areas.

\section{The case}

A 30-year-old Caucasian stay-at-home mother presented to our hospital in northern Australia with a 1-week history of undifferentiated abdominal pain and fevers. She had type 1 DM diagnosed 13 years prior, which had been managed with an insulin pump for less than 12 months. She had suboptimal control with a HbA1c of $10.1 \%$ but had no microvascular or macrovascular complications associated with her diabetes and no history of previous diabetic ketoacidosis. Her other medical history included ichthyosis vulgaris treated with acitretin, dyslipidaemia and depression. Blood cultures taken were repeatedly negative and an echocardiogram and CT scan of her chest, abdomen and pelvis were unremarkable. 
Six weeks later she developed left flank pain and rigors. On examination, she was febrile but her other vital signs were normal. Her abdomen was soft with generalised tenderness and voluntary guarding. There was pain on movement of her left hip. The skin on the plantar aspect of her feet was erythematous and desquamated, but no other skin changes were present. Her insulin pump insertion site was clean. An MRI scan revealed a left psoas abscess.

\section{Investigations}

Initial investigations revealed a normal a normal white cell $\left(4.2 \times 10^{9} / \mathrm{L}\right)$ and neutrophil $\left(3.15 \times 10^{9} / \mathrm{L}\right)$ count. Her C-reactive protein (CRP) was $70 \mathrm{mg} / \mathrm{L}$. Her glomerular filtration rate was greater than $90 \mathrm{~mL} / \mathrm{min} / 1.73 \mathrm{~m}^{2}$ with a creatinine of $32 \mu \mathrm{mol} / \mathrm{L}$. Her liver function tests were normal.

Multiple blood cultures did not grow an organism.

MRI scans are shown in Fig. 1.

As aspirate of the psoas abscess isolated Burkholderia pseudomallei.

\section{Treatment}

Drainage of the abscess was performed under CT guidance. IV ceftazidime $8 \mathrm{~g} /$ day was prescribed for 6 weeks in addition to oral trimethoprim/sulfamethoxazole 320/1600 mg (TMP/SMX) followed by 3 months of oral TMP/SMX with supplemental folic acid. She continued on her insulin pump whilst undergoing treatment.

\section{Outcome and follow-up}

Antibiotics were well tolerated with clinical and biochemical improvement monitored weekly. She had no adverse reactions to the medication. To our knowledge, there has been no evidence of relapsed infection.

\section{Discussion}

Melioidosis, caused by the environmental, Gramnegative bacterium $B$. pseudomallei, is found in the soil and surface water in tropical regions (6). The first human case of melioidosis described in Australia was in 1950, in a patient with DM from Townsville (7). In the Northern Territory of Australia the average annual incidence rate is 19.6 cases per 100,000 population, with an estimated rate in people with DM of 260 cases per 100,000/year (1).

The most common primary routes of transmission are well recognised and include direct inoculation through skin, inhalation and less commonly ingestion. Direct inoculation through abrasions in the skin is thought to be the most frequent route of acquisition $(4,8)$. Our patient had multiple possible entry points; firstly due to insulin pump insertion sites and secondly due to ichthyosis vulgaris.

Most cases of melioidosis occur in patients with underlying medical conditions (5). DM is a wellrecognised risk factor (4); a prospective study conducted over 20 years in Darwin found that 39\% of patients with melioidosis had DM with type $2 \mathrm{DM}$ occurring most commonly (1). In this study, they calculated the risk of

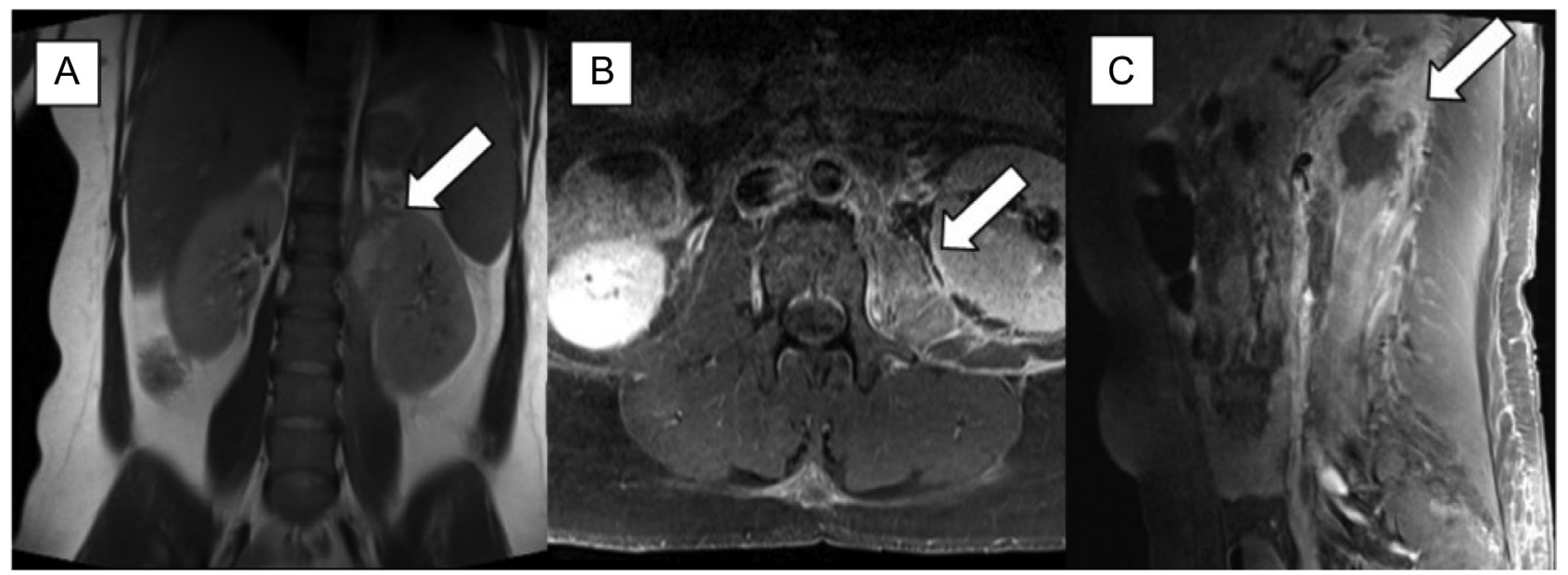

Figure 1

MRI scan of spine. A large psoas abscess is evident on (A) T2-weighted coronal view, (B) T2-weighted axial view with gadolinium and (C) T1-weighted sagittal view with gadolinium. 
melioidosis in the Northern Territory to be 21.2 times higher in patients with DM than in those without DM (1). The association with DM may be secondary to impaired neutrophil function with studies demonstrating altered chemotaxis, phagocytosis, oxidative burst and killing activity (3). A similar effect on neutrophils has been observed in other significant risk factors: heavy alcohol consumption, chronic renal failure and thalassaemia (2). Occupational exposure to $B$. pseudomallei via contact with soil and water appears to a major risk factor in Thailand with $81 \%$ of cases occurring in rural rice farmers and their families (3). In contrast, only $18 \%$ of patients in the Darwin study had an obvious occupational exposure, with most of the $49 \%$ of patients who lived in the City of Darwin acquiring the organism close to home (1).

Melioidosis usually presents acutely, with pneumonia being the most common presentation $(1,4,6)$. Fourteen percent of cases present with genitourinary infection, $13 \%$ with skin infection whilst $3.5 \%$ of patients present with soft tissue abscesses, most commonly involving the prostate, liver, spleen and kidney. Psoas abscesses are found in $<1 \%$ of cases (1). Other manifestations include septic arthritis, osteomyelitis and neurological melioidosis (1). Bacteraemia occurs in $55 \%$ of patients with $21 \%$ of patients developing septic shock which has a mortality rate of $50 \%$ (1). Chronic infection, defined by symptoms for more than 2 months, accounts for $11 \%$ of all cases of melioidosis. Symptoms can mimic those of tuberculosis with fevers, weight loss and a productive cough $(4,6)$. Patients with chronic melioidosis are less likely to have DM than those who presented with an acute illness (20 vs $42 \%$ ) (1).

In order to prevent melioidosis, patients with DM and other risk factors are encouraged to use footwear and gardening gloves in the wet season when coming in contact with soil or mud (9) and to minimise alcohol consumption (10).

In summary, melioidosis is a heterogeneous condition with protean clinical manifestations. People with DM have the greatest risk factor for disease acquisition. Whilst pulmonary disease is the most common presentation, rare presentations such as primary psoas abscess, as seen in our case, can occur. The case highlights the importance of considering atypical infections in individuals with DM even if blood cultures are negative and the need for targeted prevention in those at risk of melioidosis.
Declaration of interest

The authors declare that there is no conflict of interest that could be perceived as prejudicing the impartiality of the research reported.

\section{Funding}

This research did not relieve any specific grant from any funding agency in the public, commercial or not-for-profit sector.

\section{Patient consent}

Consent has been obtained from the patient for publication.

Author contribution statement

$M$ Katz was responsible for the case report and literature review. S Smith, L Conway and A Sinha edited the case report and literature review. A Sinha is the treating physician.

\section{References}

1 Currie BJ, Ward L \& Cheng AC. The epidemiology and clinical spectrum of melioidosis: 540 cases from the 20 year Darwin prospective study. PLOS Neglected Tropical Diseases 20104 e900. (https://doi.org/10.1371/journal.pntd.0000900)

2 Cheng A \& Currie B. Meliodosis: epidemiology, pathophysiology and management. Clinical Microbiology Reviews 200518 383-416. (https://doi.org/10.1128/CMR.18.2.383-416.2005)

3 Suputtamongkol Y, Chaowagul W, Chetchotisakd P, Lerpatanasuwun N, Intaranongpai S, Ruchutrakool T, Budhsarawong D, Mootsikapun P, Wuthiekanun V, Teerawatasook N, et al. Risk factors for melioidosis and bacteraemic meliodosis. Clinical Infectious Diseases 199929 403-413. (https://doi.org/10.1086/520223)

4 Lipsitz R, Garges S, Aurigemma R, Baccam P, Blaney DD, Cheng ACS, Currie BJ, Dance D, Gee JE, Larsen J, et al. Workshop on treatment of and postexposure prophylaxis for Burkholderia pseudomallei and B. mallei infection, 2010. Emerging Infectious Diseases 201218 e2-e2. (https://doi.org/10.3201/eid1812.120638)

5 Stewart JD, Smith S, Binotto E, McBride WJ, Currie BJ \& Hanson J. The epidemiology and clinical features of meliodosis in Far North Queensland: implications for patient management. PLOS Neglected Tropical Diseases 201711 e0005411. (https://doi.org/10.1371/journal. pntd.0005411)

6 Dance D. Treatment and prophylaxis of meliodosis. International Journal of Antimicrobial Agents 201443 310-318. (https://doi. org/10.1016/j.ijantimicag.2014.01.005)

7 Rimington RA. Meliodosis in north Queensland. Medical Journal of Australia 196249 50-53.

8 Foong YC, Tan M \& Bradbury RS. Melioidosis: a review. Rural Remote Health 2014142763.

9 Davis JS, Currie BJ, Fisher DA, Huffam SE, Anstey NM, Price RN, Krause VL, Zweck N, Lawton PD, Snelling PL, et al. Prevention of opportunistic infections in immunosuppressed patients in the tropical end of the Northern Territory. Communicable Diseases Intelligence Quarterly Report 200327 526-532.

10 Northern Territory Government. Melioidosis. Northern Territory: Northern Territory Government; 2017 [updated 2017 Nov 28; cited 2018 April 7]. Available from: https://nt.Gov.Au/wellbeing/ healthconditions-treatments/bacterial/melioidosis). 